\title{
The Recognition of Qualifications in the EU
}

Citation for published version (APA):

Kortese, L. S. J. (2020). The Recognition of Qualifications in the EU: Blurring the Lines of Competences between the Internal Market and Education. [Doctoral Thesis, Maastricht University]. Maastricht University. https://doi.org/10.26481/dis.20200528lk

Document status and date:

Published: 01/01/2020

DOI:

10.26481/dis.20200528lk

Document Version:

Publisher's PDF, also known as Version of record

\section{Please check the document version of this publication:}

- A submitted manuscript is the version of the article upon submission and before peer-review. There can be important differences between the submitted version and the official published version of record.

People interested in the research are advised to contact the author for the final version of the publication, or visit the DOI to the publisher's website.

- The final author version and the galley proof are versions of the publication after peer review.

- The final published version features the final layout of the paper including the volume, issue and page numbers.

Link to publication

\footnotetext{
General rights rights.

- You may freely distribute the URL identifying the publication in the public portal. please follow below link for the End User Agreement:

www.umlib.nl/taverne-license

Take down policy

If you believe that this document breaches copyright please contact us at:

repository@maastrichtuniversity.nl

providing details and we will investigate your claim.
}

Copyright and moral rights for the publications made accessible in the public portal are retained by the authors and/or other copyright owners and it is a condition of accessing publications that users recognise and abide by the legal requirements associated with these

- Users may download and print one copy of any publication from the public portal for the purpose of private study or research.

- You may not further distribute the material or use it for any profit-making activity or commercial gain

If the publication is distributed under the terms of Article $25 \mathrm{fa}$ of the Dutch Copyright Act, indicated by the "Taverne" license above, 


\section{Summary (EN/NL/DE/FR)}

At the basis of the recognition of qualifications is a fundamental typology that is decisive for how the topic is shaped at the European level. The typology of the recognition of qualifications is based on two objectives: recognition for the purpose of work or of studies. Whereas the former is called professional recognition, the latter is called academic recognition. Professional recognition is again divided into de jure and de facto professional recognition concerning the difference between regulated and non-regulated professions. Academic recognition can either be by substitution (i.e. concerning a student exchange programme) or by accumulation (i.e. taking full courses of study in different Member States).

This typology is not just theoretical since it has consequences for how competences are shaped in the EU Treaties. Whereas professional recognition is an EU internal market competence - and has been ever since the 1957 Rome Treaty academic recognition is placed under an education competence, which is a much younger competence originating from the 1992 Maastricht Treaty. This distinction in competences again has consequences for how recognition law and policy are shaped at the European level. Whereas the internal market competence allows the EU to harmonise national legislation and practices, the education competence expressly prohibits it. As a consequence, professional recognition is typically handled by the EU while academic recognition is handled by various actors.

In this research, the most prominent recognition instruments and initiatives adopted by various actors are the subject of analysis. When discussing efforts in this area of the EU, the Professional Qualifications Directive (2005/36/EC amended by 2013/55/EU), the Copenhagen ${ }^{2169}$ and the Sorbonne Processes ${ }^{2170}$ are included. Although these two Processes are positioned within the EU framework, they were inspired by the most famous of the processes: the Bologna Process. ${ }^{2171}$ This Process was initiated by EU Member States but is positioned outside the EU legal framework. Despite its intergovernmental (i.e. non-legally binding) character, the Bologna Process inspired the creation of the European Higher Education Area (EHEA) and harmonised the structure of higher education programmes into a three-cycle structure comprising bachelor's, master's, and doctorate degrees. Another non-EU initiative

\footnotetext{
${ }^{2169}$ Particular attention is devoted to the European Qualifications Framework for lifelong learning (EQF), the common framework for the provision of better services for skills and qualifications (Europass), the European Credit System for Vocational Education and Training (ECVET), and the European Quality Assurance Reference Framework for Vocational Education and Training (EQAVET).

2170 This Process was initiated in 2018 with the adoption of a Recommendation aiming to achieve automatic academic recognition.

${ }^{2171}$ Particular attention is given to the Qualifications Framework of the European Higher Education Area (QF-EHEA), the Diploma Supplement, the ECTS, and the Standards and Guidelines for Quality Assurance in the European Higher Education Area (ESG).
} 
included is the Council of Europe and UNESCO's Convention on the Recognition of Qualifications concerning Higher Education in the European Region (the Lisbon Recognition Convention). Lastly, this research includes two Benelux Decisions which achieved automatic mutual generic level recognition of higher education degrees.

While the Professional Qualifications Directive was adopted on the basis of the EU's internal market competence of Article 53 TFEU, the other EU instruments were all adopted on the basis of the education competence of Article 165 TFEU. The prohibition of harmonisation in that Article enables the existence of other non-EU initiatives such as the Bologna Process, Lisbon Recognition Convention, and Benelux Decisions. One may ask why it matters that we have different instruments for professional and academic recognition - are the two objectives not different, thus justifying distinct competences and instruments and initiatives?

This research explores the grey area between the areas of professional and academic recognition that challenges the harsh distinction in two recognition types. Whereas previous research identified synergies that can exist between the two areas of recognition, this research shows that the instruments and initiatives analysed also show points of overlap. For example, the Lisbon Recognition Convention and Benelux Decisions claim to focus on academic recognition, but in fact also concern professional recognition. These synergies and points of overlap therefore give rise to questions as to what the resulting European system for recognition looks like and whether such points of overlap are permitted in light of EU law.

In order to provide an answer to such questions, the interrelationships existing among the instruments and initiatives are mapped so as to construct the European system for the recognition of qualifications. An assessment regarding the coherence of the instruments and initiatives is conducted to enable potential clashes of instruments and initiatives to be brought to light. Whereas the analysis shows that there are no major incoherencies existing between the different instruments and initiatives included in this research, it also shows that points of overlap regarding the areas of professional and academic recognition are sustained by how the internal market and education competences are currently understood. Indeed, the internal market competence is understood to only concern de jure professional recognition even if nowhere in Article 53 TFEU is it stated that its scope is restricted to that subtype of recognition. The Article 165 TFEU education competence concerns academic recognition alone but has also been used to adopt transparency tools supporting any type of recognition (albeit professional or academic) in the context of the Copenhagen Process. Accordingly, the area of de facto professional recognition is not handled by the EU under the internal market competence, thus leaving it up to the Member States or other international organisations to act in this area. This situation not only gives rise to different instruments being adopted outside the EU framework, 
but also means that de facto professional recognition is pulled into the realm of academic recognition and into the scope of an education competence, whilst it is actually an internal market topic. This is unfortunate, as it sustains the existence of divergent national and international practices, increasing the complexity and pluriformity of the recognition of qualifications.

In order to demonstrate the pluriformity of recognition practices, four profession-specific case studies are included in this research. The professions included are paediatric surgeons, childcare workers, intensive care nurses, and psychologists. These case studies enable an examination as to how the European instruments and initiatives are transposed and what effects they have at the national level. In particular, the case studies in this research focus on the Netherlands, Belgium and Germany (North Rhine-Westphalia). All four case studies have a practise-based character, as they are all based on "real-life cases" researched by the author at the Institute for Transnational and Euregional cross border cooperation and Mobility / ITEM. Whereas the case studies on paediatric surgeons and childcare workers examine what challenges remain for regulated professions, the case studies regarding specialised intensive care nurses and psychologists are exemplary of the situation where recognition types overlap.

To conclude, this research shows that the recognition of qualifications is a complex, multi-faceted and fragmented topic. The large variety of legal instruments, cooperation initiatives, actors, and individuals involved has rendered the topic opaque and difficult to comprehend. The objective of this research is to provide clarity in the complex topic that is the recognition of qualifications while exploring its main actors, their legal instruments and cooperation initiatives, and the level at which they operate. Furthermore, recommendations are provided to improve the recognition of qualifications in legislation, policy, and ultimately in practice. 


\section{Samenvatting}

Het onderwerp van de erkenning van kwalificaties is gebaseerd op een fundamentele typologie die bepalend is voor de wijze waarop het onderwerp op Europees niveau wordt vormgegeven. Deze typologie is gebaseerd op twee doelstellingen: erkenning met het oog op werk of studie. Terwijl de eerste doelstelling professionele erkenning wordt genoemd, wordt de tweede doelstelling academische erkenning genoemd. Professionele erkenning is opnieuw onderverdeeld in de jure en de facto. Concreet heeft dit onderscheid betrekking op het verschil tussen gereglementeerde en nietgereglementeerde beroepen. Academische erkenning kan plaatsvinden door vervanging (d.w.z. met betrekking tot een studentenuitwisselingsprogramma) of door accumulatie (d.w.z. het volgen van een volledige studie in verschillende lidstaten).

Deze typologie is niet alleen theoretisch, aangezien zij gevolgen heeft voor de manier waarop de bevoegdheden op het gebied van erkenning in de EU-verdragen worden vormgegeven. Terwijl professionele erkenning al sinds het Verdrag van Rome (1957) een EU interne markt bevoegdheid is, wordt academische erkenning onder de onderwijsbevoegdheid geplaatst welke veel recenter is en voortvloeit uit het Verdrag van Maastricht van 1992. Dit onderscheid in bevoegdheden heeft gevolgen voor de wijze waarop wetgeving en beleid over erkenning op Europees niveau worden vormgegeven. Terwijl de bevoegdheid voor de interne markt de EU in staat stelt om nationale wet- en regelgeving te harmoniseren, is dit op grond van de onderwijsbevoegdheid uitdrukkelijk verboden. Als gevolg daarvan wordt professionele erkenning doorgaans door de EU behandeld, terwijl de academische erkenning door verschillende actoren wordt behandeld.

In dit onderzoek worden de meest prominente erkenningsinstrumenten en initiatieven van verschillende actoren geanalyseerd. Wat betreft EU gerelateerde instrumenten zijn de Richtlijn beroepskwalificaties (2005/36/EG gewijzigd door 2013/55/EU) en de Kopenhagen ${ }^{2172}$ en Sorbonne ${ }^{2173}$ processen opgenomen in dit onderzoek. Hoewel actie op het gebied van deze twee processen in het kader van de EU wordt ondernomen, zijn ze geïnspireerd door de bekendste van de processen: het Bologna proces. ${ }^{2174}$ Dit proces is geïnitieerd door de lidstaten van de EU, maar ligt

\footnotetext{
2172 Bijzondere aandacht wordt besteed aan het Europees Kwalificatieraamwerk (EQF), het gemeenschappelijk kader voor het verstrekken van betere diensten voor vaardigheden en kwalificaties (Europass), het Europees system voor studiepuntenoverdracht voor beroepsonderwijs en -opleiding (ECVET) en het Europees referentiekader voor kwaliteitsborging in beroepsonderwijs en -opleiding. ${ }^{2173}$ Dit proces is in 2018 van start gegaan met de totstandkoming van een aanbeveling gericht op het realiseren van automatische academische erkenning.

${ }^{2174}$ Bijzondere aandacht wordt besteed aan het Kwalificatieraamwerk voor de Europese Hogeronderwijsruimte (QF-EHEA), het Diploma Supplement, het Europees studiepuntensysteem (ECTS) en de Standaarden en richtlijnen voor kwaliteitszorg in de Europese Hogeronderwijsruimte (ESG).
} 
buiten het Europese rechtskader. Ondanks het intergouvernementele (d.w.z. nietjuridische) karakter van het Bologna proces heeft deze samenwerking geresulteerd in de Europese Hogeronderwijsruimte en heeft het de structuur van de hoger onderwijsopleidingen geharmoniseerd tot een driecyclusstructuur met bachelor-, master- en doctoraatsopleidingen. Een ander initiatief dat buiten het Europese rechtskader ligt is het Verdrag inzake de erkenning van getuigschriften betreffende het hoger onderwijs in de Europese regio van de Raad van Europa en UNESCO (de Lissabon Erkenningsconventie). Ten slotte bevat dit onderzoek twee Beneluxbesluiten die een automatische wederzijdse generieke niveau-erkenning van de diploma's van het hoger onderwijs hebben gerealiseerd.

Terwijl de Richtlijn beroepskwalificaties werd vastgesteld op basis van de EU interne markt bevoegdheid van artikel 53 VWEU, werden de andere EUinstrumenten vastgesteld op basis van de EU-bevoegdheid op het gebied van onderwijs van artikel 165 VWEU. Het harmonisatieverbod in dat artikel zorgt ervoor dat andere niet-EU-initiatieven, zoals het Bologna proces, de Lissabon Erkenningsconventie en de Benelux-besluiten naast de EU-wetgeving op het gebied van erkenning kunnen bestaan. Men kan zich afvragen waarom het van belang is dat we verschillende instrumenten voor professionele en academische erkenning hebben - zijn de twee doelstellingen niet verschillend, waardoor verschillende bevoegdheden en instrumenten en initiatieven gerechtvaardigd zijn?

Dit onderzoek analyseert het grijze gebied tussen professionele en academische erkenning. Dit grijze gebied zorgt ervoor dat men vraagtekens kan zetten bij het harde onderscheid in twee soorten erkenning. Terwijl uit eerder onderzoek is gebleken dat er synergieën tussen beide gebieden van erkenning kunnen bestaan, blijkt uit dit onderzoek dat de instrumenten en initiatieven die zijn opgenomen ook punten van overlap vertonen. Zo wordt in de Lissabon Erkenningsconventie en in de Benelux-besluiten de nadruk gelegd op academische erkenning, terwijl er in feite ook sprake is van professionele erkenning. Deze synergieën en punten van overlap leiden aldus tot vragen over hoe het resulterende Europese systeem voor erkenning eruitziet en of dergelijke punten van overlap zijn toegestaan in het licht van het EU-recht.

Om een antwoord te geven op dergelijke vragen worden de bestaande onderlinge verbanden tussen de instrumenten en initiatieven in kaart gebracht, zodat het Europese systeem voor de erkenning van kwalificaties kan worden opgebouwd. Er wordt een beoordeling gemaakt van de samenhang tussen de instrumenten en initiatieven om mogelijke botsingen aan het licht te brengen. Uit dit onderzoek blijkt dat er geen grote incoherenties bestaan tussen de verschillende instrumenten en initiatieven die in dit onderzoek zijn opgenomen, maar het toont ook aan dat punten van overlap op het gebied van professionele en academische erkenning in stand 
blijven door de manier waarop de interne markt en de onderwijsbevoegdheden op dit moment worden begrepen. De bevoegdheid voor de interne markt heeft immers alleen betrekking op de jure professionele erkenning - ook al wordt nergens in artikel 53 VWEU vermeld dat het toepassingsgebied ervan beperkt is tot dat subtype van erkenning. De onderwijsbevoegdheid van artikel 165 VWEU heeft alleen betrekking op academische erkenning, maar werd ook gebruikt om in het kader van het Kopenhagen proces transparantie-instrumenten vast te stellen ter ondersteuning van alle vormen van erkenning (zij het professioneel of academisch). Bijgevolg wordt het gebied van de facto professionele erkenning niet door de EU behandeld in het kader van de interne markt bevoegdheid, zodat lidstaten of andere internationale organisaties de mogelijkheid hebben om op dit gebied actie te ondernemen. Deze situatie leidt er niet alleen toe dat verschillende instrumenten buiten het kader van de EU worden aangenomen, maar betekent ook dat de facto professionele erkenning binnen de academische erkenning en de onderwijsbevoegdheid wordt getrokken, terwijl het in feite een interne markt onderwerp is. Dit is betreurenswaardig, aangezien dit het bestaan van uiteenlopende nationale en internationale praktijken in stand houdt, waardoor de complexiteit en pluriformiteit van de erkenning van kwalificaties toeneemt.

Om de veelheid van erkenningspraktijken aan te tonen, zijn in dit onderzoek vier beroepsspecifieke casestudies opgenomen. In het bijzonder gaat het om kinderchirurgen, kinderopvangmedewerkers, gespecialiseerde intensive care verpleegkundigen en psychologen. Deze casestudies maken het mogelijk om te onderzoeken hoe de Europese instrumenten en initiatieven worden omgezet en welke effecten ze hebben op nationaal niveau. De casestudies in dit onderzoek zijn gericht op Nederland, België en Duitsland (Noordrijn-Westfalen). Alle case studies hebben een praktijkgericht karakter, aangezien ze allemaal gebaseerd zijn op "real-life cases" die door de auteur zijn onderzocht bij het Institute for Transnational and Euregional cross border cooperation and Mobility / ITEM. Terwijl de casestudies over kinderchirurgen en kinderopvangmedewerkers erop gericht zijn te achterhalen welke uitdagingen nog bestaan voor gereglementeerde beroepen, zijn de casestudies over gespecialiseerde intensive care verpleegkundigen en psychologen exemplarisch voor de situatie waarin de erkenningstypes elkaar overlappen.

In conclusie, blijkt uit dit onderzoek dat de erkenning van kwalificaties een complex, veelzijdig en gefragmenteerd onderwerp is. De grote verscheidenheid aan rechtsinstrumenten, samenwerkingsinitiatieven, actoren en betrokkenen zorgen ervoor dat het onderwerp ondoorzichtig en moeilijk te begrijpen is. Het doel van dit onderzoek is dan ook om duidelijkheid te bieden over het complexe onderwerp van de erkenning van kwalificaties en tegelijkertijd de belangrijkste actoren, hun rechtsinstrumenten en samenwerkingsinitiatieven en het niveau waarop zij actief zijn 


\section{Samenvatting}

te onderzoeken. Bovendien worden aanbevelingen gedaan om de erkenning van kwalificaties in wetgeving, beleid en uiteindelijk in de praktijk te verbeteren. 


\section{Zusammenfassung}

Grundlage für die Anerkennung von Qualifikationen ist eine grundlegende Typologie, die entscheidend dafür ist, wie das Thema auf europäischer Ebene gestaltet wird. Die Typologie der Anerkennung von Qualifikationen basiert auf zwei Zielen: die Anerkennung zum Zweck der Arbeit und die Anerkennung zum Zwecke des Studiums. Während der erste Typ als berufliche Anerkennung bezeichnet wird, wird der Zweite als akademische Anerkennung bezeichnet. Im ersten Fall spricht man zudem von einer Unterteilung in eine de jure und eine de facto Form der beruflichen Anerkennung, welche den Unterschied zwischen reglementierten und nicht reglementierten Berufen widerspiegelt. Bei der akademischen Anerkennung sind wiederum die Anerkennung aufgrund von Substitution (d.h. in Bezug auf ein Studentenaustauschprogramm) oder von Akkumulation (d.h. die Teilnahme an vollständigen Studiengängen in verschiedenen Mitgliedstaaten) zu unterscheiden.

Diese Typologie ist nicht nur theoretisch, da sie konkrete Konsequenzen für die Ausgestaltung der Kompetenzen in den Verträgen der Europäischen Union (EU) hat. Während die berufliche Anerkennung der von EU und Mitgliedstaaten geteilten Zuständigkeit für den EU-Binnenmarkt zuzuordnen - und diese bereits schon seit 1957 in dem Vertrag von Rom verankert - ist, ist die akademische Anerkennung lediglich der unterstützenden Kompetenz der Union im Bildungsbereich zuzuschreiben, welche eine viel jüngere ergänzende Befugnis darstellt die dem Vertrag von Maastricht von 1992 entstammt. Diese Unterscheidung der Zuständigkeiten hat wiederum Konsequenzen für die Gestaltung des Rechtes auf Anerkennung und der Anerkennungspolitik auf europäischer Ebene. So erlaubt die Zuständigkeit für den Binnenmarkt es der EU, die nationale Gesetzgebung und Praxis $\mathrm{zu}$ harmonisieren, wohingegen die Befugnis zu Unterstützungsmaßnahmen im Bildungsbereich dies ausdrücklich verbietet. Folglich werden die Grundsätze der beruflichen Anerkennung in der Regel von der Union reguliert, während die akademische Anerkennung verschiedene Akteure involviert.

In der vorliegenden Untersuchung werden die wichtigsten Instrumente der beruflichen und akademischen Anerkennung und Initiativen verschiedener Akteure analysiert. Bei der Erörterung der Sachlage in diesem Bereich werden sowohl die EU Richtlinie über die Anerkennung von Berufsqualifikationen (2005/36/EG, geändert durch 2013/55/EU) als auch der Kopenhagener ${ }^{2175}$ und der Sorbonne ${ }^{2176}$ Prozess

\footnotetext{
2175 Besondere Aufmerksamkeit wird dem Europäischen Qualifikationsrahmen (EQR), dem gemeinsamen Rahmen für die Bereitstellung besserer Dienste für Fertigkeiten und Qualifikationen (Europass), dem Europäischen Leistungspunktesystems für die Berufsbildung (ECVET) und dem europäischen Bezugsrahmen für die Qualitätssicherung in der beruflichen Aus- und Weiterbildung (EQAVET) gewidmet.
} 
miteinbezogen. Diese beiden Prozesse sind im Rahmen der EU anzusiedeln, auch wenn sie inspiriert wurden durch den wohl bekanntesten dieser sogenannten Prozesse: den Bologna Prozess. ${ }^{2177}$ Letzterer wurde von den EU-Mitgliedstaaten initiiert, ist aber außerhalb des EU-Rechtsrahmens angesiedelt. Trotz seines intergouvernementalen (d.h. nicht rechtsverbindlichen) Charakters inspirierte der Bologna Prozess die Schaffung des Europäischen Hochschulraums (EHEA) und harmonisierte die Struktur der Hochschulstudiengänge in eine dreistufige Struktur, die Bachelor-, Master- und Doktorats-Abschlüsse umfasst. Eine weitere Nicht-EUInitiative betrifft das gemeinsame Übereinkommen von Europarat und UNESCO über die Anerkennung von Qualifikationen im Hochschulbereich in der europäischen Region (das sogenannte Lissabonner Anerkennungsübereinkommen). Darüber hinaus umfasst diese Studie auch zwei Beschlüsse der Benelux Union, mit denen die automatische gegenseitige allgemeine Niveauanerkennung von Hochschulabschlüssen erreicht wurde.

Während die Berufsqualifikationsrichtlinie auf der Grundlage der Binnenmarktkompetenz der EU nach Artikel 53 AEUV verabschiedet wurde, basieren die übrigen EU-Instrumente alle auf der Grundlage der Unterstützungsbefugnis im Bildungsbereich nach Artikel 165 AEUV. Das Harmonisierungsverbot in diesem letzteren Artikel ist die Voraussetzung für die Existenz anderer Nicht-EU-Initiativen wie den Bologna Prozess, und lässt auch dem Lissabonner Anerkennungsübereinkommen und den Benelux-Beschlüssen mehr Gewicht zukommen. Man kann sich fragen, warum es wichtig ist, dass wir unterschiedliche Instrumente für die berufliche und akademische Anerkennung haben. Rechtfertigen die beiden unterschiedlichen Ziele der beruflichen und akademischen Anerkennung nicht auch die Existenz unterschiedlicher Kompetenzen, Instrumenten und Initiativen?

Diese Studie zeigt, dass zwischen den Bereichen der beruflichen und akademischen Anerkennung eine Grauzone besteht, die die harte Unterscheidung zwischen zwei Anerkennungstypen in Frage stellt. Während frühere Forschungen Synergien zwischen den beiden Bereichen identifiziert haben, zeigt diese Studie, dass die Instrumente und Initiativen, die aufgenommen sind, auch Überschneidungen aufweisen. So geben beispielsweise das Lissabonner Anerkennungsübereinkommen und die Benelux-Beschlüsse vor, sich auf die akademische Anerkennung zu konzentrieren, betreffen aber tatsächlich auch die berufliche Anerkennung. Diese

\footnotetext{
${ }^{2176}$ Der Sorbonne Prozess wurde in 2018 mit einer Empfehlung des Rates der EU eingeleitet, die auf eine automatische akademische Anerkennung abzielt.

2177 Besondere Aufmerksamkeit wird den Qualifikationsrahmen für den Europäischen Hochschulraum (QF-EHEA), den Diplomzusatz, das europäische System zur Übertragung und Akkumulierung von Studienleistungen (ECTS) und den Standards und Leitlinien für die Qualitätssicherung im Europäischen Hochschulraum (ESG) gewidmet.
} 
Synergien und Überschneidungen werfen daher die Frage auf, was für ein europäisches Anerkennungssystem eigentlich daraus resultiert und ob solche Überschneidungen im Lichte des EU-Rechts zulässig sind.

Um eine Antwort auf solche Fragen zu geben, werden die zwischen den Instrumenten und Initiativen bestehenden Wechselbeziehungen inventarisiert, um das europäische System zur Anerkennung von Qualifikationen abzubilden. Folglich wird die Kohärenz der Instrumente und Initiativen bewertet, um mögliche Kollisionen der Instrumente und Initiativen zu identifizieren. Während die Untersuchung einerseits zeigt, dass es keine großen Inkohärenzen zwischen den verschiedenen Instrumenten und Initiativen gibt, die in diese Untersuchung einbezogen wurden, zeigt sie andererseits auch, dass das derzeitige Verständnis des Binnenmarktes und der Unterstützungskompetenzen im Bildungsbereich die Überschneidungen in den Bereichen der beruflichen und akademischen Anerkennung tatsächlich aufrechterhalten. Tatsächlich wird die Binnenmarktkompetenz so verstanden, dass sie nur die de jure Form der beruflichen Anerkennung betrifft - auch wenn nirgendwo in Artikel 53 AEUV festgehalten ist, dass ihr Anwendungsbereich auf diese Unterart der Anerkennung beschränkt ist. Die Bildungskompetenz nach Artikel 165 AEUV zielt dahingegen formell auf die akademische Anerkennung ab, wurde allerdings auch dazu verwendet, um im Rahmen des Kopenhagen Prozesses Transparenzinstrumente $\mathrm{zu}$ verabschieden, die jede Art der Anerkennung (beruflich oder akademisch) unterstützen. Dementsprechend wird der Bereich der de facto beruflichen Anerkennung tatsächlich nicht von der EU im Rahmen der Binnenmarktkompetenz behandelt, wodurch es den Mitgliedstaaten oder anderen internationalen Organisationen überlassen bleibt, in diesem Bereich tätig zu werden. Diese Situation führt nicht nur dazu, dass verschiedene Instrumente außerhalb des EU-Rahmens angenommen werden, sondern bedeutet auch, dass die de facto berufliche Anerkennung in den Bereich der akademischen Anerkennung und die Bildungskompetenz gezogen wird, obwohl sie eigentlich ein Thema des Binnenmarktes ist. Dies ist bedauerlich, da es die Existenz unterschiedlicher nationaler und internationaler Praktiken aufrechterhält, was die Komplexität und Pluralität der Anerkennung von Qualifikationen erhöht.

Um die Pluralität der Anerkennungspraktiken zu demonstrieren, werden vier berufsspezifische Fallstudien in diese Forschung einbezogen. Die einbezogenen Berufe sind Kinderchirurgen, Erzieher, Intensivpfleger und Psychologen. Diese Fallstudien ermöglichen eine Analyse, wie die europäischen Instrumente und Initiativen umgesetzt werden und welche Auswirkungen sie auf nationaler Ebene haben. Die vorliegenden Fallstudien konzentrieren sich geografisch insbesondere auf die Niederlande, Belgien und Deutschland (Nordrhein-Westfalen). Alle vier Fallstudien haben einen praxisbezogenen Charakter, da sie alle auf "realen Fällen" 
basieren, die die Autorin am Institute for Transnational and Euregional cross border cooperation and Mobility / ITEM recherchiert hat. Während die Fallstudien über Kinderchirurgen und Erzieher untersuchen, welche Herausforderungen für die reglementierten Berufe noch bestehen, sind die Fallstudien über Intensivpfleger und Psychologen beispielhaft für die Situation, in der sich die Anerkennungsarten überschneiden.

Im Fazit zeigt diese Studie, dass die Anerkennung von Qualifikationen ein komplexes, facettenreiches und fragmentiertes Thema ist. Die große Vielfalt an Rechtsinstrumenten, Kooperationsinitiativen, Akteuren und beteiligten Personen hat das Thema undurchsichtig und schwer verständlich gemacht. Ziel dieser Forschung ist es, Klarheit über das komplexe Thema der Anerkennung von Qualifikationen zu schaffen und gleichzeitig die Hauptakteure, ihre Rechtsinstrumente und Kooperationsinitiativen sowie die Ebene, auf der sie operieren, zu untersuchen. Darüber hinaus werden Empfehlungen zur Verbesserung der Anerkennung von Qualifikationen in der Gesetzgebung, der Politik und schließlich in der Praxis gegeben. 


\section{Résumé}

À la base de la reconnaissance des qualifications se trouve une typologie fondamentale qui est déterminante pour la façon dont le sujet est façonné au niveau européen. La typologie de la reconnaissance des qualifications est basée sur deux objectifs : la reconnaissance à des fins de travail ou d'études. Alors que le premier est appelé reconnaissance professionnelle, le second est appelé reconnaissance académique. La reconnaissance professionnelle est à nouveau divisée en reconnaissance professionnelle de jure et de facto concernant la différence entre les professions réglementées et non réglementées. La reconnaissance académique peut se faire soit par substitution (c'est-à-dire concernant un programme d'échange d'étudiants), soit par accumulation (c'est-à-dire en suivant des cours complets d'études dans différents États membres).

Cette typologie n'est pas seulement théorique car elle a des conséquences sur la façon dont les compétences sont façonnées dans les traités de l'UE. Alors que la reconnaissance professionnelle est une compétence du marché intérieur de l'UE - et ce depuis le traité de Rome de 1957 - la reconnaissance académique est placée sous une compétence en matière d'éducation, qui est une compétence beaucoup plus jeune issue du traité de Maastricht de 1992. Cette distinction des compétences a à nouveau des conséquences sur la manière dont le droit et la politique de reconnaissance sont façonnés au niveau européen. Alors que la compétence relative au marché intérieur permet à l'UE d'harmoniser les législations et les pratiques nationales, la compétence en matière d'éducation l'interdit expressément. Par conséquent, la reconnaissance professionnelle est généralement gérée par l'UE tandis que la reconnaissance académique est gérée par différents acteurs.

Dans cette recherche, les principaux instruments de reconnaissance et les initiatives adoptées par les différents acteurs font l'objet d'une analyse. Lors de l'examen des efforts déployés dans ce domaine au sein de l'UE, la directive relative aux qualifications professionnelles (2005/36/CE modifiée par 2013/55/UE), le processus de Copenhague ${ }^{2178}$ et le processus de Sorbonne ${ }^{2179}$ sont inclus. Bien que ces deux processus se situent dans le cadre de l'UE, ils ont été inspirés par le plus célèbre des processus : le processus de Bologne. ${ }^{2180}$ Ce processus a été initié par les États

\footnotetext{
${ }^{2178}$ Une attention particulière est accordée au cadre européen des certifications pour l'apprentissage tout au long de la vie (CEC), au cadre commun pour l'offre de meilleurs services dans le domaine des aptitudes et des certifications (Europass), au système européen de crédit d'apprentissages pour l'enseignement et la formation professionnels (ECVET) et au cadre européen de référence pour l'assurance de la qualité dans l'enseignement et la formation professionnels (EQAVET).

${ }^{2179}$ Ce processus a été lancé en 2018 avec l'adoption d'une recommandation visant à réaliser une reconnaissance académique automatique,

${ }^{2180}$ Une attention particulière est accordée au Cadre de l'Espace européen de l'enseignement supérieur (QF-EHEA), au supplément au diplôme, au Système européen de transfert et d'accumulation de crédits
} 


\section{Résumé}

membres de l'UE, mais il est positionné en dehors du cadre juridique de l'UE. Malgré son caractère intergouvernemental (c'est-à-dire non juridiquement contraignant), le processus de Bologne a inspiré la création de l'Espace Européen de l'Enseignement Supérieur (EEES) et a harmonisé la structure des programmes d'enseignement supérieur en une structure à trois cycles comprenant des diplômes de licence, de maîtrise et de doctorat. Une autre initiative non européenne concerne la Convention du Conseil de l'Europe et de l'UNESCO sur la reconnaissance des qualifications relatives à l'enseignement supérieur dans la région européenne (la Convention de reconnaissance de Lisbonne). Enfin, cette recherche inclut deux décisions du Benelux qui ont permis la reconnaissance mutuelle automatique générique de niveau des diplômes de l'enseignement supérieur.

Alors que la directive relative aux qualifications professionnelles a été adoptée sur la base de la compétence de l'UE en matière de marché intérieur de l'article 53 TFUE, les autres instruments de l'UE ont tous été adoptés sur la base de la compétence en matière d'éducation de l'article 165 TFUE. L'interdiction d'harmonisation prévue par cet article permet l'existence d'autres initiatives non européennes telles que le processus de Bologne, la Convention de reconnaissance de Lisbonne et les décisions Benelux. On peut se demander pourquoi il est important que nous ayons des instruments différents pour la reconnaissance professionnelle et académique - les deux objectifs ne sont-ils pas différents, justifiant ainsi des compétences, des instruments et des initiatives distinctes?

Cette recherche explore la zone grise entre les domaines de la reconnaissance professionnelle et académique qui remet en cause la distinction entre les deux types de reconnaissance. Alors que des recherches antérieures ont identifié des synergies qui peuvent exister entre les deux domaines de reconnaissence, cette recherche montre que les instruments et les initiatives analysés présentent également des points de chevauchement. Par exemple, la Convention de reconnaissance de Lisbonne et les décisions Benelux déclarent se concentrer sur la reconnaissance académique, mais concernent en fait aussi la reconnaissance professionnelle. Ces synergies et ces points de chevauchement soulèvent donc des questions quant à la forme du système européen de reconnaissance qui en résulte et quant à savoir si ces points de chevauchement sont autorisés à la lumière du droit communautaire.

Afin de répondre à ces questions, les interrelations qui existent entre les instruments et les initiatives sont cartographiées de manière à construire le système européen de reconnaissance des qualifications. Une évaluation de la cohérence des instruments et des initiatives est réalisée pour permettre de mettre en évidence d'éventuels conflits entre les instruments et les initiatives. Si l'analyse montre qu'il

(ECTS) et aux Références et lignes directrices pour l'assurance qualité dans l'espace européen de l'enseignement supérieur (ESG). 
n'existe pas d'incohérences majeures entre les différents instruments et initiatives inclus dans cette recherche, il montre également que les points de chevauchement qui concernent les domaines de la reconnaissance professionnelle et académique sont soutenus par la manière dont le marché intérieur et les compétences en matière d'éducation sont actuellement compris. En effet, la compétence du marché intérieur est comprise comme ne concernant que la reconnaissance professionnelle de jure même si nulle part dans l'article 53 TFUE il est indiqué que son champ d'application est limité à ce sous-type de reconnaissance. La compétence en matière d'éducation de l'article 165 du TFUE ne concerne que la reconnaissance académique, mais a également été utilisée pour adopter des outils de transparence soutenant tout type de reconnaissance (professionnelle ou académique) dans le contexte du processus de Copenhague. En conséquence, le domaine de la reconnaissance professionnelle de facto n'est pas traité par l'UE dans le cadre de la compétence du marché intérieur, laissant ainsi aux États membres ou à d'autres organisations internationales le soin d'agir dans ce domaine. Cette situation non seulement donne lieu à l'adoption de différents instruments en dehors du cadre de l'UE, mais signifie également que la reconnaissance professionnelle de facto est intégrée dans le domaine de la reconnaissance académique et dans le champ d'application de la compétence en matière d'éducation, alors qu'il s'agit en fait d'un sujet relevant du marché intérieur. Cette situation est regrettable, car elle entretient l'existence de pratiques nationales et internationales divergentes, ce qui accroît la complexité et la pluriformité de la reconnaissance des qualifications.

Afin de démontrer la multitude des pratiques de reconnaissance, quatre études de cas spécifiques à la profession sont incluses dans cette recherche. Les professions concernées sont les chirurgiens pédiatriques, les éducateurs, les infirmières en soins intensifs et les psychologues. Ces études de cas permettent d'examiner comment les instruments et initiatives européens sont transposés et quels effets ils ont au niveau national. Les études de cas de cette recherche portent notamment sur les Pays-Bas, la Belgique et l'Allemagne (Rhénanie-du-Nord-Westphalie). Les quatre études de cas ont un caractère pratique, car elles sont toutes basées sur des "cas réels" étudiés par l'auteur à l'Institute for Transnational and Euregional cross border cooperation and Mobility / ITEM. Alors que les études de cas sur les chirurgiens pédiatriques et les éducateurs examinent les défis qui restent à relever pour les professions réglementées, les études de cas concernant les infirmières en soins intensifs et les psychologues spécialisés en soins intensifs sont exemplaires de la situation où les types de reconnaissance se chevauchent.

Pour conclure, cette recherche montre que la reconnaissance des qualifications est un sujet complexe, multiforme et fragmenté. La grande variété d'instruments juridiques, d'initiatives de coopération, d'acteurs et d'individus 
impliqués a rendu le sujet opaque et difficile à comprendre. L'objectif de cette recherche est de clarifier le sujet complexe de la reconnaissance des qualifications tout en explorant ses principaux acteurs, leurs instruments juridiques et leurs initiatives de coopération, ainsi que le niveau auquel ils opèrent. En outre, des recommandations sont fournies pour améliorer la reconnaissance des qualifications dans la législation, la politique et, finalement, dans la pratique. 